\title{
MS06-04 | Molecular Mechanisms of TREX1 In DNA Repair ANd Immune Silencing
}

Huang, Kuan-Wei (Department of Biological Science and Technology, National Chiao-Tung university, Hsinchu, TWN); Liu, TungChang (Institute of molecular medicine and bioengineering, National Chiao-Tung university, Hsinchu, TWN); Hsiao, Yu-Yuan (Department of Biological Science and Technology, National Chiao-Tung university, Taiwan, Hsinchu, TWN); Hsaio, Yu-Yuan (Institute of molecular medicine and bioengineering, National Chiao-Tung university, Hsinchu, TWN)

Mammalian TREX1 provides the major $3^{\prime}$-to- $5^{\prime}$ exonuclease activity in immune silencing and DNA repair, and its defects are related to multiple inflammation and autoimmune disorders. A long-standing mystery is how TREX1 conducts $3^{\prime}$-overhang excision in DNA repair and dsDNA degradation in immune silencing. Here, the molecular basis of such unique catalytic activities are established by crystal structure analyses of TREX1 bound with a variety of structural DNA bearing a short 3'-overhang or damaged nucleotides. We found that the Leu24-Pro25-Ser26 cluster serves to cap the non-scissile $5^{\prime}$-end of the DNA for precise removal of the $3^{\prime}$-overhang or it wedges into the duplex region for dsDNA degradation. We also revealed the behaviours of TREX1 in acting on DNA lesions in the various DNA repair pathways and cooperating with HMGB-2 in immune silencing. This study thus uncovered unprecedented knowledge on the molecular mechanism of TREX1 in DNA processing. 